\title{
Los fármacos más consumidos en personas mayores con estado nutricional deteriorado
}

\author{
L. Regueiro-Folgueira, A. Maseda, L. Lorenzo-López, A. Buján, I. González- \\ Abraldes, J.C. Millán-Calenti
}

Universidade da Coruña, Grupo de Investigación en Gerontología, Instituto de Investigación Biomédica de A Coruña (INIBIC), Complexo Hospitalario Universitario de A Coruña (CHUAC), SERGAS, A Coruña, España

Objetivos: Los cambios nutricionales influyen sobre la farmacocinética y la farmacodinámica de los medicamentos consumidos, pudiendoademáslos medicamentos implicar déficits nutricionales. Mediante el presente trabajo pretendemos determinar los fármacos más consumidos en un grupo de personas mayores no institucionalizadas de acuerdo a su estado nutricional.

Método: Se realizó un estudio transversal con una muestra representativa de la población mayor gallega. Participaron 749 personas de 65 o más años, residentes en sus domicilios y usuarios de asociaciones de personas mayores. Se llevó a cabo una valoración geriátrica integral que incluía, entre otras, la valoración del estado nutricional mediante el Mini Nutritional Assesment- Short Form (MNA-SF) y la recogida de las prescripciones médicas mediante presentación de la historia farmacológica, usando para su categorización la clasificación anatómica, terapéutica y química (ATC).

Resultados: Se observaron diferencias estadísticamente significativas entre el número de prescripciones médicas y el estado nutricional de los sujetos. La media diaria de consumo de fármacos en personas con una nutrición deficiente fue de 7,14. Los fármacos más consumidos entre las personas con bajas puntuaciones en el MNA-SF - y por tanto con un estado nutricional deficiente-, fueron los indicados para el sistema cardiovascular $(76,6 \%)$, y dentro de estos, los más utilizados fueron los agentes modificadores de lípidos $(50,5 \%)$. El segundo puesto lo formaron los medicamentos pertenecientes al grupo del tracto alimentario y metabolismo (70,1\%), abundando aquí los relativos a los trastornos de acidez (48,6\%). El tercer puesto lo ocuparon los destinados al sistema nervioso $(68,2 \%)$, y dentro de estos los más comunes fueron los psicolépticos $(47,7 \%)$.

Conclusiones: Un estado nutricional deficiente se asocia con una elevada polifarmacia, siendo los fármacos destinados al sistema cardiovascular, al tracto alimentario y metabolismo y al sistema nervioso los más utilizados. Las personas mayores deberían concienciarse de la necesidad de promover tratamientos no farmacológicos, cambiando su estilo de vida y mejorando su estado nutricional.

Este trabajo ha sido financiado por la Xunta de Galicia (ED431C2017/49 y Red FrailNet IN607C 2016/08). 